\title{
64. Exponential Decrease of Susceptibility to Anaesthesia with Age in the Water-flea, Moina macrocopa Strauss."
}

By Arata Terao and Tomozô Tanaka.

(Comm. by C. IshikawA, M.I.A., May 12, 1930.)

Previously ${ }^{2)}$ we have called attention to the age factor in the time required to anaesthetize the water-flea, Moina macrocopa Strauss. The purpose of the present paper is to analyse mathematically our data which are reproduced here with some additional remarks.

A Senô-Tauti serial incubator ${ }^{3}$ was divided into three compartments for hatching, keeping, and rearing the water-flea. These ran at $34^{\circ}$, $27^{\circ}$, and $20^{\circ} \mathrm{C}$. respectively. A single parthenogenetic female was first put in a jar in the rearing compartment with water which contained a large number of unicellular algae, Scenedesmus, as food and this was changed daily. About two weeks later a numerous population had grown in this jar, and this culture was kept going in a good condition by changing the water daily and removing enough of the cladocerans so that there was no over-crowding.

Experimental animals were obtained by selecting from the culture a number of well-fed females with embryos ready to hatch and putting them in a jar in the hatching compartment at $34^{\circ} \mathrm{C}$. for an hour (from noon to 1 o'clock P. M.) with a view to accelerating the hatching. The young individuals thus obtained were designated as of zero age, and either subjected at once to experimentation or kept in vials in the stock compartment $\left(27^{\circ} \mathrm{C}\right.$.) for further experiment in relation to with the daily change of the culture medium. A $\mathrm{m} / 50$ solution of chloretone warmed in a water-bath, the temperature of which was maintained at $27^{\circ} \mathrm{C}$., was put in test-tubes which were also kept in the water-bath. The experimental animals were dropped in the solution contained in these test-tubes, where they finally turned on their backs

1) Contributions from Zoological Laboratory, Imperial Fisheries Institute, Tokyo, No. 6 (Preliminary note with same title was published in Jour. Imp. Fish. Inst. 25 (3) : 63-64 1 Fig. 1930).

2) Terao, A. and T. Tanaka, Anaesthesia of the water-flea, Moina macrocopa Strauss, in relation to age. Jour. Imp. Fish. Inst. (Tokyo) 25 (1): 13-14. 1 Fig. 1929.

3) We are much indebted to the staff of our Physical Laboratory, especially to Professor M. Tauti, for help in the regulation of this apparatus. 
as they lost their power of maintaining balance. This act was taken as a sign of anaesthesia and time required for it to take place was determined with stopwatches in $1 / 5$ second units. The length of the carapace of each individual tested was also measured under the microscope. The biometric constants are given in Table 1 , and the mean values of time required for anaesthesia and those of carapace length are plotted with relation to age in Figs. 1 and 2 .

Table 1. Age, carapace length, and time required to anaesthetize the water-flea, Moina macrocopa Strauss.

\begin{tabular}{r|c|c|c|c|c|c|c}
\hline $\begin{array}{c}\text { Age } \\
\text { in } \\
\text { days }\end{array}$ & $\begin{array}{c}\text { Number } \\
\text { of in. } \\
\text { dividuals }\end{array}$ & \multicolumn{2}{|c|}{ Time required for anaesthesia, in seconds } & \multicolumn{3}{c}{ Carapace length in millimeters } \\
\hline & & Mean & $\begin{array}{c}\text { Standard } \\
\text { deviation }\end{array}$ & $\begin{array}{c}\text { Coefficient of } \\
\text { variation }\end{array}$ & Mean & $\begin{array}{c}\text { Standard } \\
\text { deviation }\end{array}$ & $\begin{array}{c}\text { Coefficient } \\
\text { of variation }\end{array}$ \\
\hline 0 & 30 & $64.67 \pm 1.03$ & $8.33 \pm 0.73$ & $12.88 \pm 1.14$ & $.5300 \pm .0653$ & $.0435 \pm .0038$ & $8.21 \pm .72$ \\
1 & 25 & $205.00 \pm 3.41$ & $25.30 \pm 2.41$ & $12.34 \pm 1.20$ & $.8970 \pm .1210$ & $.0402 \pm .0038$ & $4.48 \pm .43$ \\
2 & 26 & $306.15 \pm 3.57$ & $27.01 \pm 2.53$ & $8.82 \pm .83$ & $1.0365 \pm .1371$ & $.0288 \pm .0027$ & $2.78 \pm .26$ \\
3 & 25 & $342.80 \pm 10.73$ & $79.57 \pm 7.59$ & $23.21 \pm 2.33$ & $1.1630 \pm .1569$ & $.0382 \pm .0036$ & $3.23 \pm .31$ \\
4 & 25 & $275.00 \pm 11.84$ & $87.77 \pm 8.37$ & $31.92 \pm 3.91$ & $1.1890 \pm .1604$ & $.0301 \pm .0029$ & $2.53 \pm .24$ \\
5 & 25 & $269.80 \pm 13.19$ & $97.74 \pm 9.32$ & $36.47 \pm 3.91$ & $1.1910 \pm .1607$ & $.0809 \pm .0077$ & $6.80 \pm .65$ \\
6 & 25 & $418.40 \pm 9.56$ & $70.86 \pm 6.76$ & $16.94 \pm 1.66$ & $1.2850 \pm .1733$ & $.0367 \pm .0035$ & $2.86 \pm .27$ \\
7 & 25 & $476.60 \pm 12.20$ & $90.41 \pm 8.62$ & $18.97 \pm 1.85$ & $1.4810 \pm .1998$ & $.0516 \pm .0049$ & $3.48 \pm .33$ \\
8 & 25 & $483.00 \pm 10.98$ & $81.39 \pm 7.76$ & $16.85 \pm 1.65$ & $1.3730 \pm .1852$ & $.0360 \pm .0034$ & $2.62 \pm .25$ \\
9 & 24 & $360.00 \pm 13.65$ & $99.12 \pm 9.65$ & $27.53 \pm 2.88$ & $1.3646 \pm .1879$ & $.0816 \pm .0079$ & $5.98 \pm .58$ \\
10 & 27 & $390.74 \pm 7.01$ & $54.02 \pm 4.04$ & $13.83 \pm 1.11$ & $1.3898 \pm .1804$ & $.0299 \pm .0027$ & $2.15 \pm .20$ \\
\hline
\end{tabular}

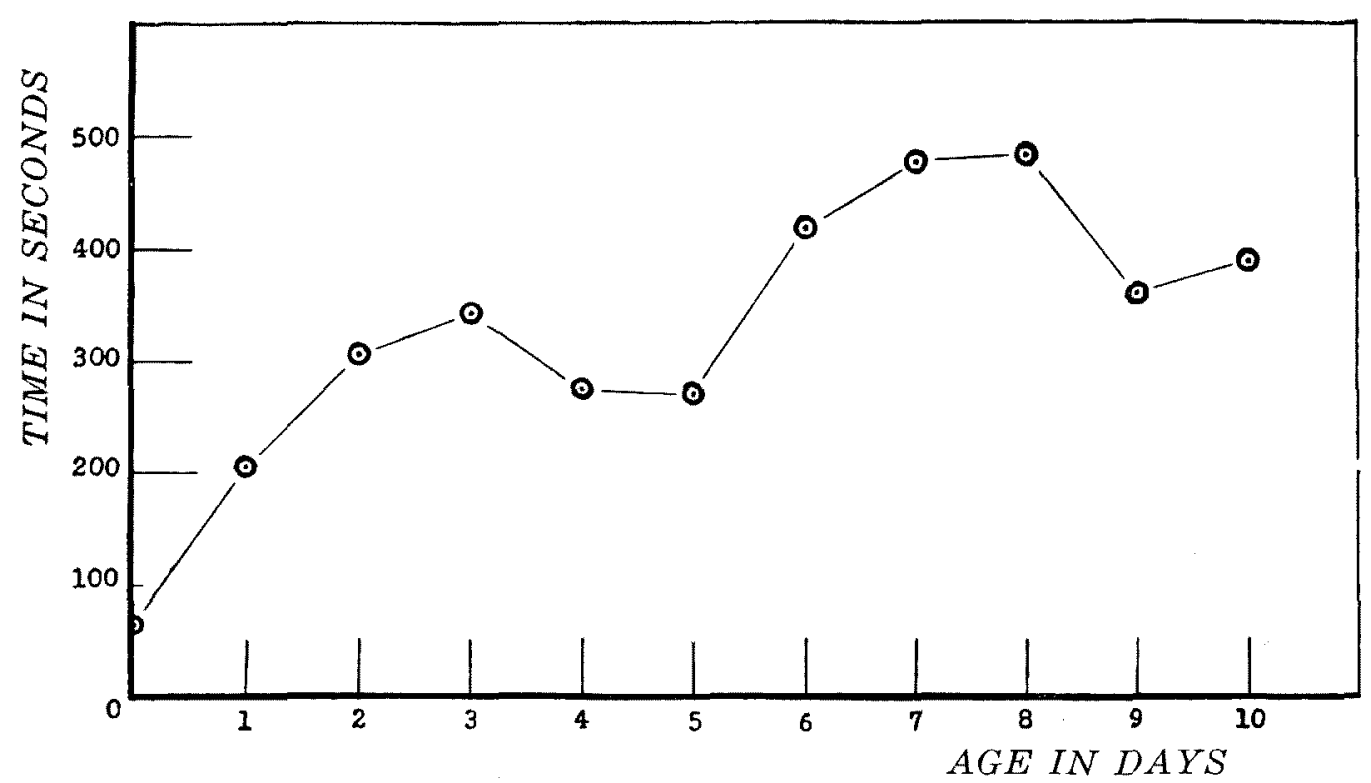

Fig. 1. Showing time in seconds required to anaesthetize Moina macrocopa Strauss in relation to age (in days). 


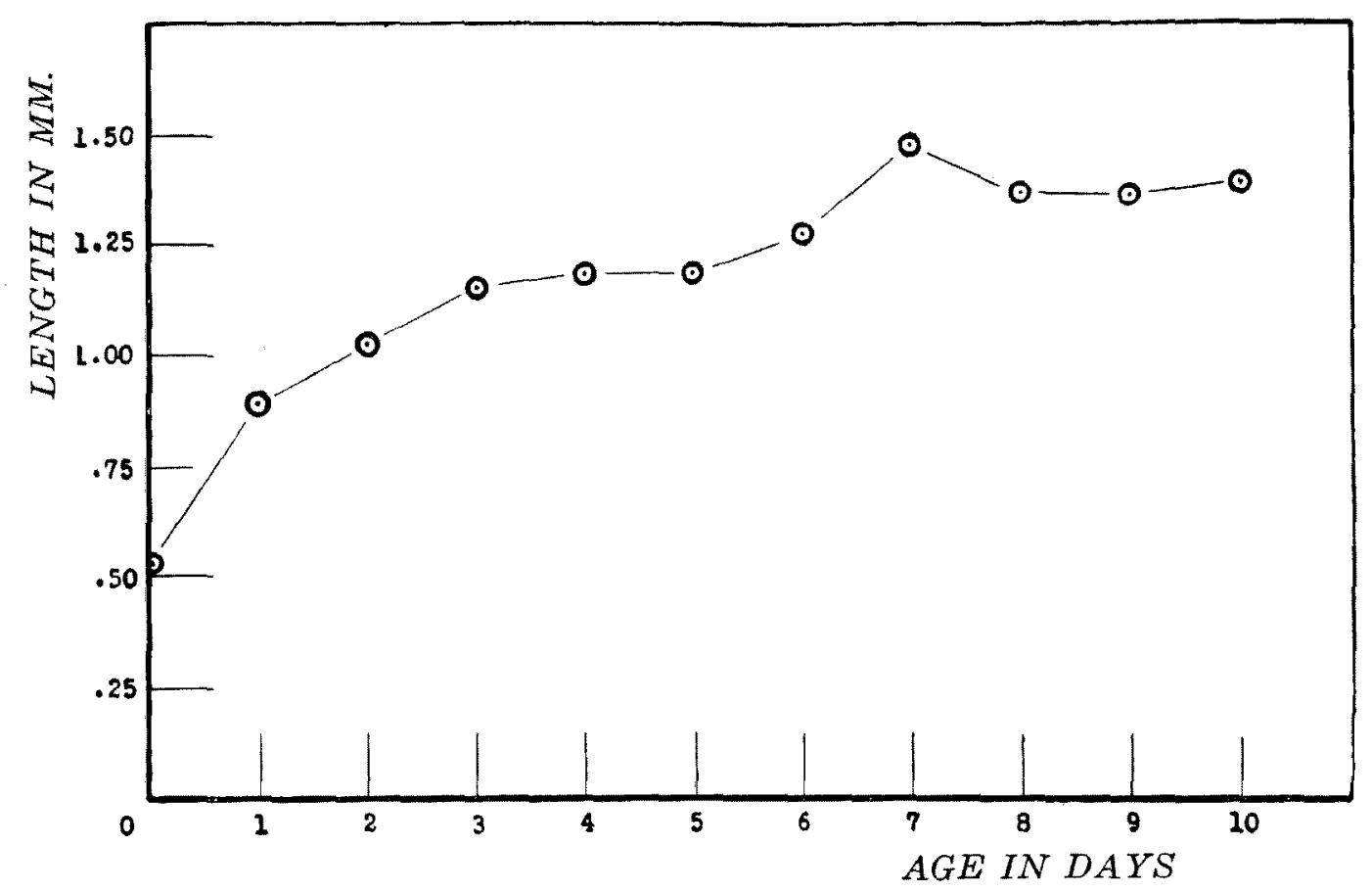

Fig. 2. Showing growth of carapace length in $\mathrm{mm}$. of Moina macrocopa Strauss.

It may be mentioned that the mean value of carapace lengths for water-fleas seven days old (Fig. 3) is larger than that of those for individuals eight days old. But this is simply due to a sampling error since we see that the square root of the sum of the squares of the probable errors of the two means is nearly the same as the difference of the two means. On the other hand, the two drops in the curve which represents time required for anaesthesia on the basis of age (Fig. 1) are clearly not due to sampling errors. Roughly speaking, time required to anaesthetize a water-flea increases as it grows older, the general trend being parabolic. But this does not mean anything, inasmuch as there is growth in size of the experimental animals and this changes the net relation between age and time required for anaesthesia.

For mathematical analysis of our data four points may be considered. First, the velocity of anaesthesia in a lot of animals of this type is proportional to the ratio of surface area to volume of the body, provided other conditions are equal. Second, this ratio is given in terms of the reciprocal of the body length among individuals of a geometrically similar shape. Third, our experimental animals reach sexual maturity, when reared at a constant temperature of $27^{\circ} \mathrm{C}$., 
two days after hatching and maintain from this stage on a nearly similar shape. Fourth, the carapace length is a rather reliable measure of the body length of our material.

It follows therefore that a series of the ratios of the mean values of time required for anaesthesia to that of the carapace length of sexually mature age groups must represent susceptibility of the waterflea to anaesthesia in relation to age. Remarkably enough, this series shows linearity when plotted on an arithrolog grid as in Fig. 3.

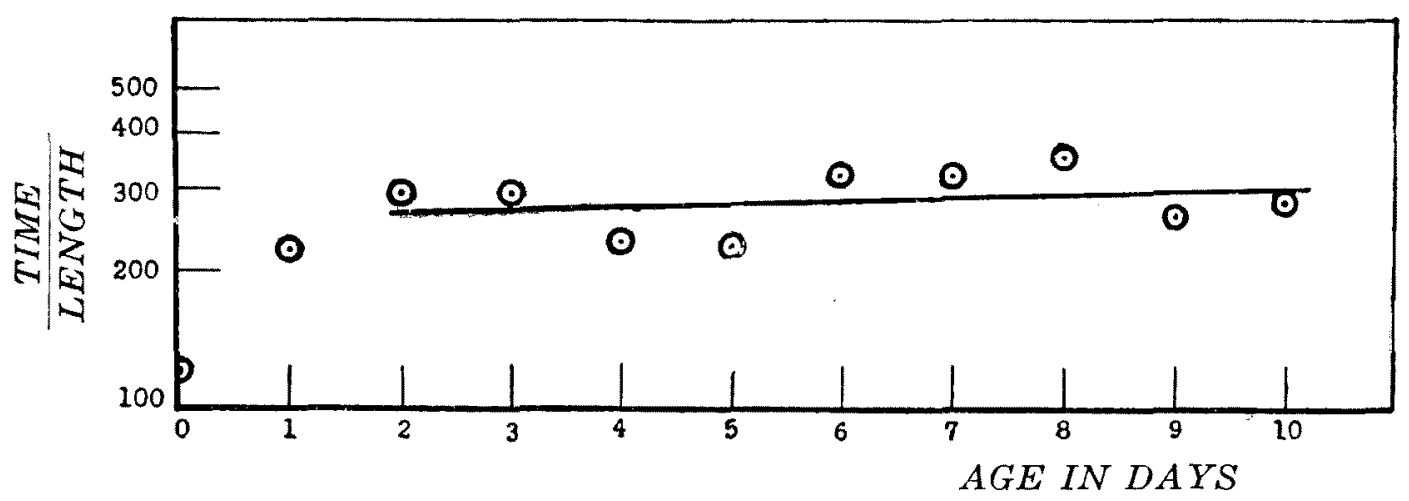

Fig. 3. Showing the relation between age (in days) and ratio of time (in seconds) required for anaesthesia to carapace length (in $\mathrm{mm}$.) of Moina macrocopa Strauss. Arithrolog grid.

Therefore it may be said in a general way that in these populations susceptibility decreases exponentially as age increases. Computing the values of the constants by the method of least squares we have

$$
y=266.962 e^{.011 x}
$$

in which $y$ denotes the ratio of time required for anaesthesia to the carapace length in sec.-mm. units, $x$ age in days, and $e$ base of Naperian logarithm.

It may be argued that such decrease is due to the change of vitality with age as was dicussed in the dairy cow and the domestic fowl by Brody and his collaborators ${ }^{1)}$ who also resorted to exponential formulae. But it should be borne in mind that our results are based on the ratios of surface area to volume of the body of the age populations and are not of the same nature as those of the abovementioned investigators.

1) Brody, S., A. C. Ragsdale, and C. W. Turner, The rate of growth of the dairy cow. III. The relation between growth in weight and increase of milk secretion with age. Jour. Gen. Physiol. 6 (1): 21-30. 3 Fig. 1923. IV. Growth and senescence as measured by the rise and fall of milk secretion with age. Ditto, 31-40 3 Fig. 1923. Brody, S., E. W. Earl, and H. L. Kempster, The rate of senescence of the domestic fowl as measured by the decline in egg production with age. Ditto, 41-45. 1 Fig. 1923. 